\title{
Spectrum of the Hermitian Wilson Dirac operator
}

\section{FSU-SCRI-98C-88}

\author{
Rajamani Narayanan ${ }^{\text {a }}$ \\ a SCRI, The Florida State University, Tallahassee, FL 32306-4130, USA \\ Recent results on the spectral properties of the Hermitian Wilson-Dirac operator are presented.
}

\section{Introduction}

Continuum gauge field theory works under the assumption that all fields are smooth functions of space time. This assumption is certainly a valid one for quantum gauge field theories that respect gauge invariance: One should always be able to fix a gauge so that the gauge fields are smooth functions of space time since the action that contains derivatives in gauge fields will not allow it otherwise. The space of smooth gauge fields typically has an infinite number of disconnected pieces with the number of pieces being in one to one correspondence with the set of integers [1]. Every gauge field in each piece can be smoothly interpolated to another gauge field in the same piece but there is no smooth interpolation between gauge fields in different pieces. This is the case for $\mathrm{U}(1)$ gauge fields in two dimensions and $\mathrm{SU}(\mathrm{N})$ gauge fields in four dimensions.

In lattice gauge theory, gauge fields are represented by link variables $U_{\mu}(x)$ that are elements of the gauge group. Continuum derivatives are replaced by finite differences and the concept of smoothness of gauge fields does not apply. Any lattice gauge field configuration, $U_{\mu}(x)=e^{i A_{\mu}(x)}$ can be deformed to the trivial gauge field configuration by the interpolation $U_{\mu}(x ; \tau)=e^{i \tau A_{\mu}(x)}$ with $U_{\mu}(x ; 1)=U_{\mu}(x)$ and $U_{\mu}(x ; 0)=1$. Since smoothness does not hold on the lattice away from the continuum limit, the space of gauge fields on the lattice forms a simply connected space. Separation of the gauge field space into an infinite number of disconnected pieces can only be realized in the continuum limit.

In this talk, I will address the following basic question: Do we see a separation of lattice gauge fields configurations into topological classes as we approach the continuum limit? To answer this question, I will assume that an ensemble of lattice gauge field configurations are given to me. This could be a pure gauge field ensemble or an ensemble that includes fermion dynamics using either staggered fermions or Wilson fermions. My aim is to answer this question without altering the lattice gauge field configuration. I will use a Wilson-Dirac fermion to probe the lattice gauge field configuration. My motivation is the overlap formalism [2] for chiral gauge theories. Topological aspects of the background gauge field are properly realized by the chiral fermions in this formalism and therefore it provides a good framework to answer the above question. The hermitian Wilson Dirac operator enters the construction of lattice chiral fermions in the overlap formalism and topological properties of the gauge fields are studied by looking at the spectral flow of the hermitian Wilson Dirac operator as a function of the fermion mass.

The talk is organized as follows. I begin by explaining the connection between the spectral flow of the hermitian Wilson Dirac operator and the topological content of the background gauge field. I then present possible scenarios for the qualitative nature of the spectrum on the lattice. I will then present some properties of the spectral flow, and I will illustrate these properties by studying the spectral flow in a single instanton background. I will then focus on the spectral properties on lattice gauge field ensembles and their behavior as the continuum limit is approached. I will only present an overview of the results. Detailed re- 
sults can be found in the talk presented by Edwards [3].

\section{Spectral flow, topology and condensates}

The massless Dirac operator in the continuum anticommutes with $\gamma_{5}$. Therefore, the non-zero imaginary eigenvalues of the massless Dirac operator come in pairs, $\pm i \lambda$ with $\psi$ and $\gamma_{5} \psi$ being the two eigenvectors. The zero eigenvalues of the massless Dirac operator are also eigenvalues of $\gamma_{5}$. These chiral zero modes are a consequence of the topology of the background gauge field. It is useful to consider the spectral flow of the Hermitian Dirac operator:

$\mathrm{H}(m)=\gamma_{5}\left(\gamma_{\mu} D_{\mu}+m\right)$

The non-zero eigenvalues of the massless Dirac operator combine in pairs to give the following eigenvalue equation:

$\mathrm{H}(m) \chi_{ \pm}= \pm \sqrt{\lambda^{2}+m^{2}} \chi_{ \pm}$

$\chi_{ \pm}$are linear combinations of $\psi$ and $\gamma_{5} \psi$ and these modes never cross the $\mathrm{x}$-axis in the spectral flow of $\mathrm{H}(m)$ as a function of $m$. The zero eigenvalues, $\gamma_{\mu} D_{\mu} \phi_{ \pm}=0$ with $\gamma_{5} \phi_{ \pm}= \pm \phi_{ \pm}$result in

$\mathrm{H}(m) \phi_{ \pm}= \pm m \phi_{ \pm}$

These modes associated with topology result in flow lines that cross the x-axis. A positive slope corresponds to positive chirality and vice-versa. The net number of lines crossing zero (the difference of positive and negative crossings) is the topology of the background gauge field. Global topology of gauge fields cause exact zero eigenvalues at $m=0$. In addition, one can have a non-zero spectral density at zero. At infinite volume in the continuum, the spectrum is continuous and $\rho(\lambda ; m) d \lambda$ is the number of eigenvalues in the infinitesimal region $d \lambda$. The spectral gap $\lambda_{g}(m)$ defined as the lowest eigenvalue at $m$ is equal to $|m|$. The spectral density at zero, $\rho(0 ; m)$ can be non-zero only at $m=0$ indicating chiral symmetry breaking in a theory like QCD.

To study the possible emergence of the above continuum picture as a continuum limit of a lattice gauge theory picture, we need to have a lattice realization of $\mathrm{H}(m)$. It is important to note

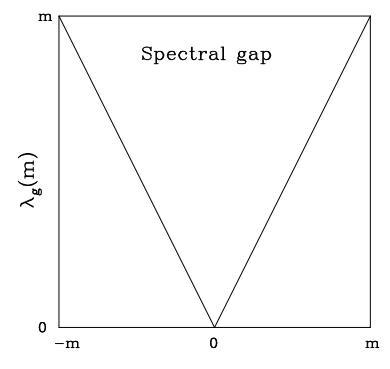

m

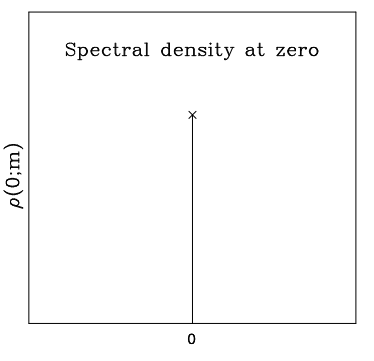

$\mathrm{m}$
Figure 1. Continuum picture of the spectral gap and the spectral density at zero.

that we are interested in the spectral flow of a single Dirac fermion. With this in mind, we choose the hermitian Wilson-Dirac operator obtained by multiplying the standard Wilson-Dirac operator by $\gamma_{5}$ :

$\mathrm{H}_{L}(m)=\left(\begin{array}{cc}\mathrm{B}(U)+m & \mathrm{C}(U) \\ \mathrm{C}^{\dagger}(U) & -\mathrm{B}(U)-m\end{array}\right)$.

$\mathrm{C}$ is the naive lattice first derivative term and $\mathrm{B}$ is the Wilson term. We are interested in the spectral flow of $\mathrm{H}_{L}(m)$ as a function of $m$. We note that $m=0,-2,-4,-6,-8$ are the points where the free fermions become massless with degeneracies $1,4,6,4,1$ respectively. Next we observe that $\mathrm{H}_{L}(m)$ can have a zero eigenvalue only if $m<0$ [4]. Let $\left(\begin{array}{l}u \\ v\end{array}\right)$ be a normalized eigenvector of $\mathrm{H}_{L}(m)$ with zero eigenvalue. Then $m u+\mathrm{B} u+\mathrm{C} v=0$ and $\mathrm{C}^{\dagger} u-\mathrm{B} v-m v=0$. This implies that $u^{\dagger} \mathrm{B} u+v^{\dagger} \mathrm{B} v=-m$. Since $\mathrm{B}$ is a positive definite operator, the above equation can have a solution only if $m<0$.

We focus on the range $-2 \leq m \leq 0$ and propose the following scenarios for the spectral gap and the spectral density at zero on the lattice and their approach to the continuum limit. Six different scenarios are possible as shown in Fig. 2.

- On the lattice we have (a) and (i) with $m_{c} \rightarrow 0$ in the continuum limit. $\rho(0 ; 0)$ approaches the continuum limit with proper 
Spectral Gap

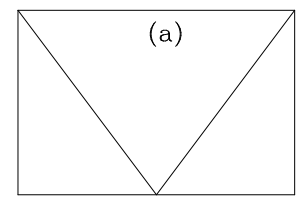

$m_{c}$
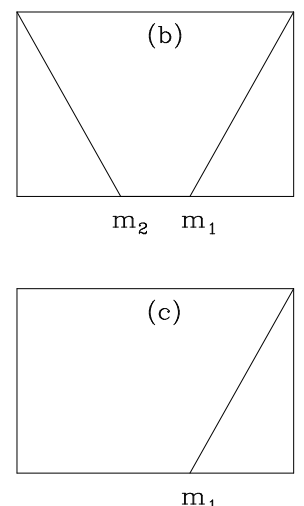

Spectral density at zero

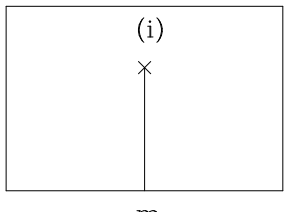

$m_{c}$

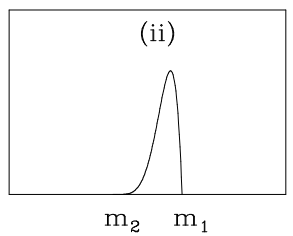

(iii)

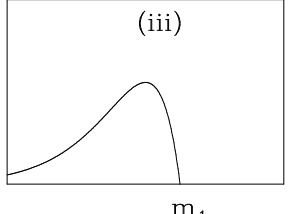

Figure 2. Possible scenarios of the spectral gap and the spectral density at zero on the lattice.

scaling taken into account.

- On the lattice we have (b) and (ii) with (a) and (i) being the continuum limit. In this case, $m_{1} \rightarrow 0$ and $m_{2} \rightarrow 0$. In the limit we also get $\rho(0 ; 0)$.

- On the lattice we have (c) and (ii) with (a) and (i) being the continuum limit. In this case, $m_{1} \rightarrow 0$. The gap opens up for $m<0$ and again we get $\rho(0 ; 0)$.

- On the lattice we have (c) and (ii) with (c) and (i) being the continuum limit. In this case, $m_{1} \rightarrow 0$. But the gap does not open up for $m<0$.

- On the lattice we have (c) and (iii) with (a) and (i) being the continuum limit. In this case, $m_{1} \rightarrow 0$. The gap opens up for $m<0$ and again we get $\rho(0 ; 0)$.

- On the lattice we have (c) and (iii) with (c) and (i) being the continuum limit. Here also $m_{1} \rightarrow 0$ and $\rho(0, m)=0$ if $m<0$. But the gap does not open up for $m<0$.

I will show that numerical studies of the spectral flow on various ensembles favor the last scenario. This seems to be the case for any lattice gauge ensemble whether the ensemble was generated with fermion dynamics or not. One should keep in mind that the Wilson fermion used to the study the spectral flow is only a probe and does not enter the dynamics that generated the gauge field ensemble.

\section{Properties of the lattice spectral flow}

I now present a topological argument which will show that zero eigenvalues of $\mathrm{H}_{L}(m)$ can occur anywhere in the region $-8<m<0$ [5]. The spectrum of $\mathrm{H}_{L}(m)$ and $-\mathrm{H}_{L}(-8-m)$ are identical for an arbitrary gauge field background. Since zero eigenvalues can occur only for $m<0$ in $\mathrm{H}_{L}(m)$, it follows that zero eigenvalues can occur only in the region $-8<m<0$. It also follows that every level crossing zero from above in the spectral flow of $\mathrm{H}_{L}(m)$ should be accompanied by a level crossing zero from below. In a smooth instanton background a level crossing zero from above at $m_{1}$ is accompanied by another level crossing zero from below at $m_{2}<m_{1}$. The second crossing is due to one of the four doubler modes. Both $m_{1}$ and $m_{2}$ will be functions of the size of the instanton $\rho$ in lattice units. For $\rho>>a, m_{1} \approx 0$ and $m_{2} \approx-2$. As $\rho$ decreases, $m_{1}$ moves farther away from zero and $m_{2}$ moves away from -2 and closer to $m_{1}$. This motion as a function of $\rho$ is smooth and for some value of $\rho, m_{1}=m_{2}$. Spectral flow changes smoothly as the configuration is slowly changed. As we move in configuration space the topological charge of a configuration changes. Tracing the spectral flow as a function of configurations shows that zero eigenvalues of $\mathrm{H}_{L}(m)$ can occur anywhere in the region $-8<m<0$.

Fig. 3 illustrates the various properties of the spectral flow of the Hermitian Wilson-Dirac operator. The background gauge field is a smooth instanton with $\rho=2.0$ on a $8^{4}$ lattice. The first crossing on the right of the figure from above to below is the one associated with the particle feeling the global topology of the gauge field. Then 


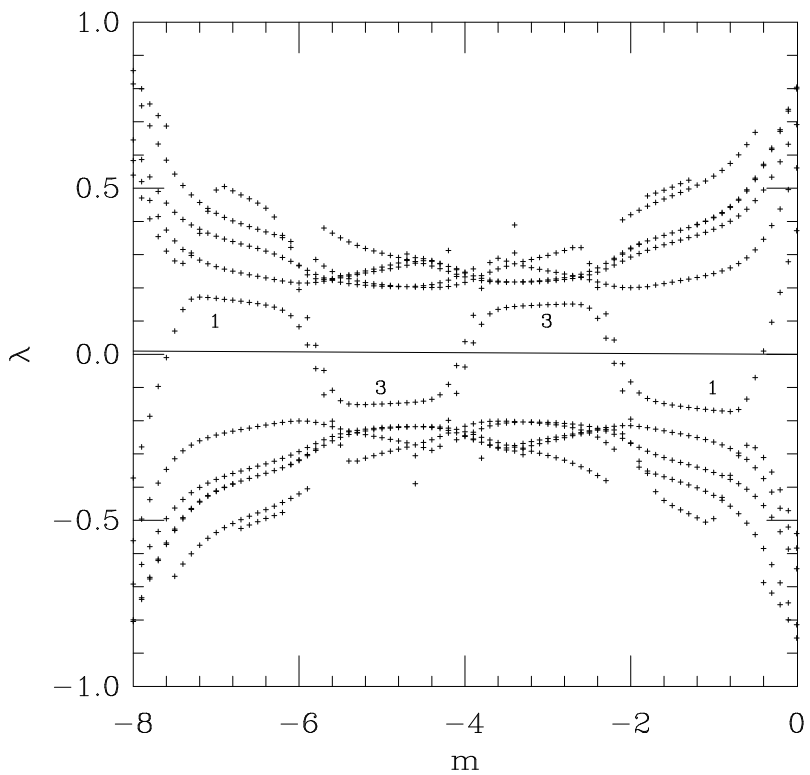

Figure 3. Spectral flow of a smooth SU(2) instanton with $\rho=2.0$ on an $8^{4}$ lattice

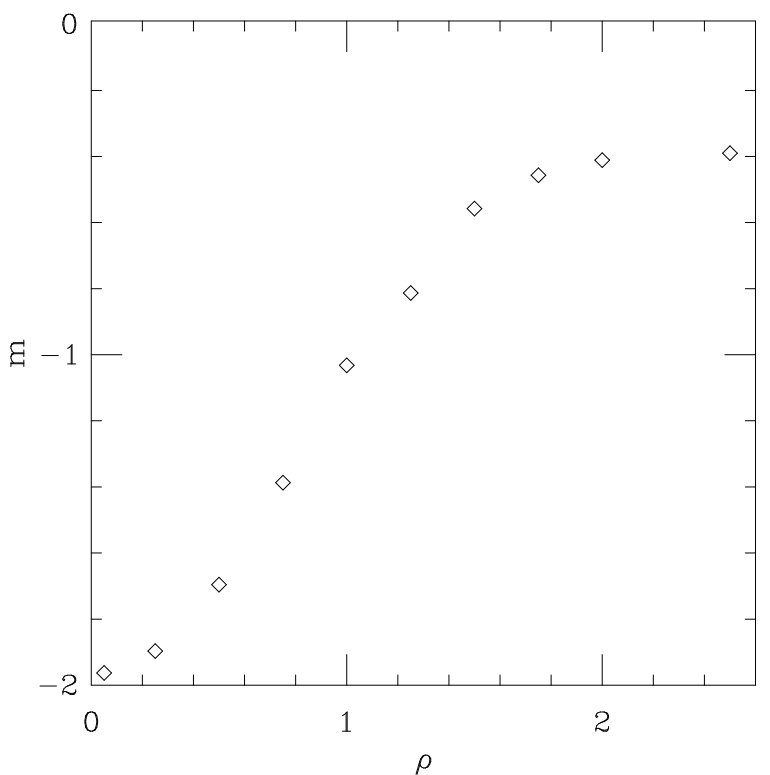

Figure 4. $m_{1}$ versus $\rho$ for a smooth instanton there are four levels crossing zero out of which three are degenerate. The degeneracy is a consequence of the smoothness of the lattice instanton. The four crossings are attributed to the four doublers feeling the global topology. This is followed by six levels crossing zero, four levels crossing zero and finally one level crossing zero. Clearly the spectrum of $\mathrm{H}_{L}(m)$ and $-\mathrm{H}_{L}(8-m)$ are the same. There is no net level crossing in the range $-8 \leq m \leq 0$. This is because the chiralities of the sixteen particles are paired. Fig. A shows the location of the first level crossing zero as one varies the size of the instanton in lattice units. As the size of the instanton is decreased the crossing point moves farther away from zero. $\rho<1$ instantons are placed on the lattice by centering the instanton on a lattice site. This figure shows that one can move the first crossing all the way up to $m=-2$.

\section{Spectral density at zero}

In the previous section, I argued that $\mathrm{H}_{L}(m)$ can have zero crossings anywhere in the region $-2 \leq m \leq 0$. Therefore the spectral gap is zero in this region on the lattice. This has direct implications for how the spectral density at zero behaves on the lattice. A careful study of the spectral density at zero has been performed on a variety of $\mathrm{SU}(3)$ pure gauge ensembles [6]. This is done by computing the low lying eigenvalues of $\mathrm{H}_{L}(m)$ using the Ritz functional [7]. The low lying eigenvalues over the whole ensemble is then used to obtain the integral of the spectral density function, namely $\int_{0}^{\lambda} \rho\left(\lambda^{\prime} ; m\right) d \lambda^{\prime}$. This is then fitted to a polynomial and $\rho(0 ; m)$ is obtained as the coefficient of the linear term in the fit. All ensembles show a peak in $\rho(0 ; m)$ at some value of $m$. There is a sharp rise to the peak from the right and a gradual fall from the peak on the left. There is a gradual rise again to a second peak at the location of the first set of doublers. The peak itself gets sharper as one goes toward the continuum limit. It also moves to the right. $\rho(0 ; m)$ is non-zero for $-2 \leq m \leq m_{1}$ in the infinite volume limit at any finite value of the lattice gauge coupling. $m_{1}$ goes to zero as the lattice coupling approaches the continuum. $\rho(0 ; m)$ approaches 


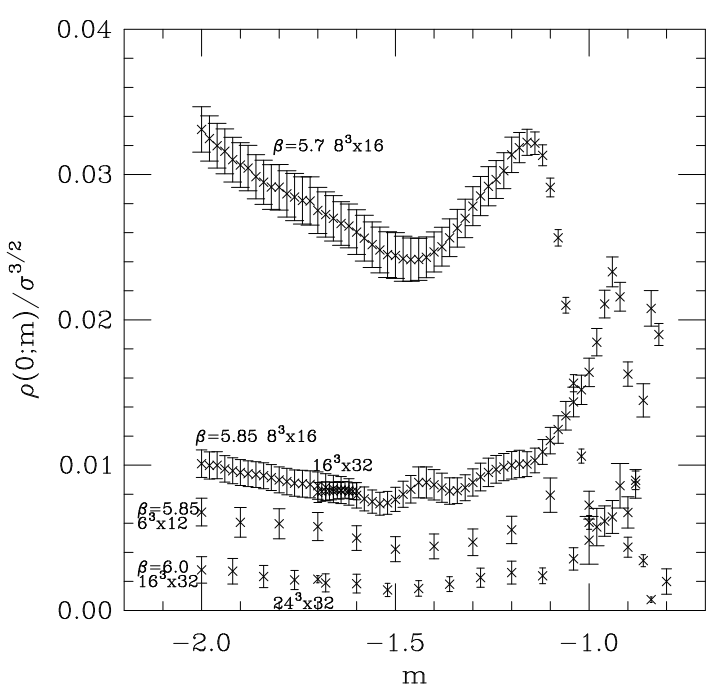

Figure 5. $\rho(0 ; m)$ as a function of $m$ for various $\mathrm{SU}(3)$ pure gauge ensembles.

the infinite lattice volume limit from below as expected. We are fairly confident that we have the infinite volume limit estimate for $\rho(0 ; m)$ at all the lattice spacings plotted in Fig. 5. In Fig. 6 , I focus on the behavior of $\rho(0 ; m)$ at a fixed $m$ as one approaches the continuum limit. The first figure indicates that $\rho(0 ; m)$ goes to zero exponentially in the inverse lattice spacing. This is given some credence by plotting the same figure in a logarithmic scale. The function seems to favor a fit of the form $b e^{-c / \sqrt{a}}$. The power of $a$ in the exponent is a consequence of an emperical fit but the data presents substantial evidence for the following: $\rho(0 ; m)$ is non-zero for all finite lattice spacings. The approach to zero at zero lattice spacing is faster than any power of the lattice spacing. This shows that the last scenario presented in the previous section is favored by the numerical results on the lattice.

\section{Discussion}

A probe of lattice gauge fields using Wilson fermions has revealed that fields are not continuum like on the lattice at gauge couplings that are typically considered to be weak. If they were continuum like, we should have seen evidence that $\rho(0, m)$ is non-zero at a single value of $m$ or a region in $m$ that is of the order of the lattice spacing. Further we should have seen a symmetry in the spectrum at values of $m$ on either side of

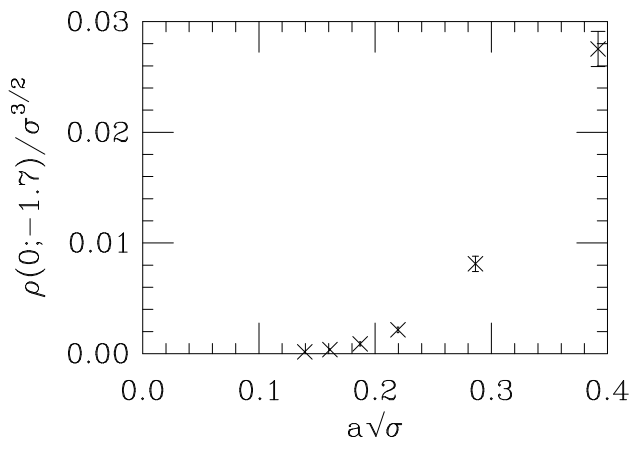

5

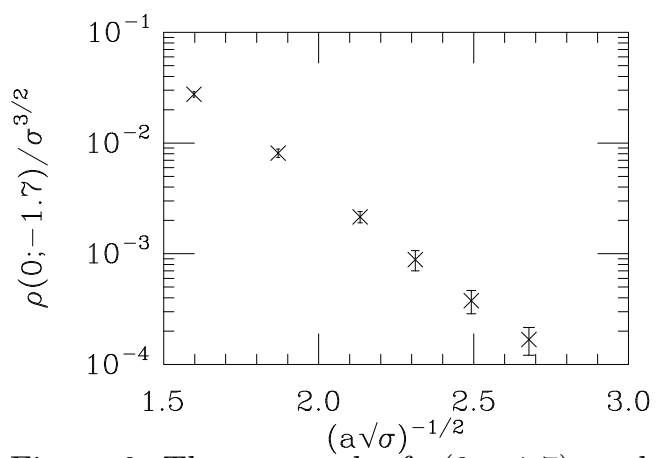

Figure 6 . The approach of $\rho(0 ;-1.7)$ to the continuum limit

the point (or region) where $\rho(0, m)$ is non-zero. Instead, we found that $\rho(0, m)$ is non-zero in a region $-2 \leq m \leq m_{1}$. In the continuum limit, there is evidence that $m_{1}$ goes to zero and that $\rho(0, m)$ goes to zero away from $m=0$. But the spectral distribution does not show evidence for a symmetry as $m \rightarrow-m$.

In addition to studying $\rho(0, m)$, one should also look at the density of levels crossing zero in an infinitesimal range $d m$ centered at $m$. In the continuum we expect level crossings only at $m=0$ but we find a finite density of level crossings zero where ever $\rho(0 ; m)$ is non-zero [3]. This implies that the topological charge of a lattice gauge field configuration defined as the net level crossings in $\mathrm{H}_{L}(m)$ in the range $[m, 0]$ will depend on $m$. The topology of a single lattice gauge field configuration is not interesting in a field theoretic sense. One has to obtain an ensemble average of the topological susceptibility and study its dependence on $m$. This has been studied on a variety of ensembles [3.6] and the results show that the topological susceptibility is essentially independent of $m$ in the region to the left of the peak in $\rho(0 ; m)$. This indicates that the levels that cross 
zero to the left of the peak are not physically relevant consistent with the result that $\rho(0 ; m)$ goes to zero in the continuum limit. The numbers for the topological susceptibility obtained in this manner are consistent with the results obtained by field theoretic methods [8]. The modes that cross zero to the left of the peak in $\rho(0 ; m)$ correspond to small modes [6,3] and this is consistent with Fig. 4 .

A remark on the approach of $\rho(0, m)$ to the thermodynamic limit at a fixed lattice gauge coupling is in order. For definiteness, let us consider a pure gauge ensemble. If the extent of the lattice is smaller than the extent needed for a finite temperature phase transition then $\rho(0, m)$ will be essentially zero. One will see a sharp increase in $\rho(0, m)$ at lattice volumes close to the phase transition. A table of $\beta_{c}\left(N_{\tau}\right)$ can be found in [9] for $\mathrm{SU}(3)$ and $\mathrm{SU}(2)$. For example, one needs to have a lattice volume bigger than $8^{4}$ if one is using an $\mathrm{SU}(3)$ gauge coupling of $\beta=6.0$ and one needs to work with a lattice larger than $14^{4}$ at $\beta=6.38$. Only then will one see a value of $\rho(0, m)$ close to the thermodynamic limit.

Studies of the spectrum of the Wilson-Dirac operator is not in any sense a new field of research. Smit and Vink [10] studied the complex spectrum of the Wilson-Dirac operator to understand topology. These initial studies led to the understanding of dislocations in lattice field configurations that affect the field theoretic determination of topological charge. Spectral flows of the hermitian Wilson Dirac operator were also studied to obtain some understanding of the role of topology in the mesonic spectrum in quenched QCD [11]. Distribution of real eigenvalues of the massless Wilson-Dirac operator (identical to the zero eigenvalues of the Hermitian Wilson-Dirac operator) has received a lot of attention recently motivated by the need to understand the inability in extracting pion masses close to the chiral limit in quenched QCD [12].

\section{Acknowledgments}

I would like to thank my collaborators, Robert Edwards and Urs Heller. All work I have done on the spectrum of the Hermitian Wilson-Dirac operator has been in collaboration with them. I would also like to thank Pavlos Vranas and Herbert Neuberger for useful discussions. This research was supported by DOE contracts DEFG05-85ER250000 and DE-FG05-96ER40979.

\section{REFERENCES}

1. T. Eguchi, P.B. Gilkey and A.J. Hanson, Phys. Rep. 66 (1980) 213.

2. R. Narayanan and H. Neuberger, Nucl. Phys. B443 (1995) 305.

3. R.G. Edwards, U.M. Heller and R. Narayanan these proceedings.

4. R. Narayanan and H. Neuberger, Phys. Rev. Lett 71 (1993) 3251.

5. R.G. Edwards, U.M. Heller and R. Narayanan Nucl. Phys. B522 (1998) 285.

6. R.G. Edwards, U.M. Heller, R. Narayanan hep-lat/9802016, To appear in Nuclear Physics B.

7. B. Bunk, K.Jansen, M. Lüscher and H. Simma, DESY Report (September 1994); T. Kalkreuter and H. Simma, Comput. Phys. Commun. 93 (1996) 33.

8. B. Alles, M. D'Elia and A. Di Giacomo, Nucl. Phys. B494 (1997) 281. T. DeGrand, Anna Hasenfratz, Tamas Kovacs, hep-lat/9801037; P. de Forcrand, M.G. Perez, J.E. Hetrick, I-O. Stamatescu, hep-lat/9802017.

9. J. Fingberg, U. Heller and F. Karsch, Nucl. Phys. B392 (1993) 493.

10. J. Smit and J. Vink, Nucl. Phys. B286 (1987) 485.

11. S. Itoh, Y. Iwasaki, T. Yoshie, Phys. Rev. D36 (1987) 527.

12. W. Bardeen, A. Duncan, E. Eichten, and H. Thacker, hep-lat/9806002; W. Bardeen, A. Duncan, E. Eichten, G. Hockney and H. Thacker,Phys. Rev. $D 57$ (1998) 1633; Nucl. Phys. Proc. Suppl. B63 (1998) 141; C.R. Gattringer, I. Hip and C.B. Lang, Nucl. Phys. Proc. Suppl. 63 (1998) 498; C.R. Gattringer and I. Hip, this proceedings, heplat/9712015; P. Hernandez, hep-lat/9801035. R.G. Edwards, U.M. Heller, R. Narayanan and R.L. Singleton, Jr, Nucl. Phys. B518 (1998) 319. 